Halimuddin Sawali, MBBS, MSurg (ORL-HNS)' Mohd Razif Mohamad Yunus, MBBS, MSurg (ORL-HNS)2 Ong Cheng Ai, MBBS, MSurg (ORL-HNS)' Primuharsa Putra Sabir Husin Athar, MD, MSurg (ORL-HNS) ${ }^{3}$
'Department of Otorhinolaryngology

Head \& Neck Surgery

Queen Elizabeth Hospital

Kota Kinabalu, Sabah, Malaysia

${ }^{2}$ Department of Otorhinolaryngology Head \& Neck Surgery

Universiti Kebangsaan Malaysia Medical Centre

Kuala Lumpur, Malaysia

${ }^{3}$ Ear, Nose, Throat-Head \& Neck Consultant Clinic KPJ Seremban Specialist Hospital

Seremban, Negeri Sembilan, Malaysia
Correspondence: Primuharsa Putra Sabir Husin Athar, MD, MSurg Consultant ENT-Head \& Neck Surgeon,

KPJ Seremban Specialist Hospital,

Jalan Toman 1, Kemayan Square, 70200 Seremban, Negeri Sembilan,

Malaysia

Fax: (606) 7653406

E-mail : putrani@yahoo.co.uk

Reprints will not be available from the authors.

The authors declared that this represents original material that is not being considered for publication or has not been published or accepted for publication elsewhere, in full or in part, in print or electronic media; that the manuscript has been read and approved by all the authors, that the requirements for authorship have been met by each author, and that each author believes that the manuscript represents honest work;

Disclosures: The authors signed disclosures that there are no financial or other (including personal) relationships, intellectual passion, political or religious beliefs, and institutional affiliations that might lead to a conflict of interest.

Presented at the 4th International Federation of Head \& Neck Oncologic Societies (IFHNOS) World Congress, Lotte Hotel, Seoul, South Korea, 15-19 June, 2010.

\title{
Cutaneous Metastases from Nasopharyngeal Carcinoma: A Rare Manifestation
}

\begin{abstract}
Objectives: In this article, we report on two unusual cases of advanced nasopharyngeal
\end{abstract} carcinoma (NPC) with distant cutaneous metastases.

\section{Methods:}

\author{
Design: Case Report \\ Setting: Tertiary Referral Center \\ Patients: Two
}

Results: Two patients with advanced NPC developed multiple nodular skin metastases, one after completing radiotherapy and another during concurrent chemo-radiotherapy. Biopsies of these skin lesions confirmed metastatic NPC and both patients succumbed to the disease.

Conclusion: Nasopharyngeal carcinoma with skin metastases carries a very poor prognosis. Early detection, diagnosis and treatment are still the best management strategy for nasopharyngeal carcinoma.

Keywords: Nasopharynx, nasopharyngeal carcinoma, skin metastases, cutaneous metastases

Nasopharyngeal carcinoma (NPC) has a higher risk of distant metastases than other malignant head and neck tumours. Together with lymph node involvement, the high rate of proliferation could account for its propensity to develop distant metastases. The incidence of isolated distant metastasis is high, globally at $18 \%$, and the risk increases with the stage of the disease ( $47 \%$ for stage IVB), demonstrating that locoregional treatment alone for locally advanced disease is inadequate. 'The lung is the most common site of metastasis, followed by bone and the liver. ${ }^{2}$ However, skin metastases in NPC patients are extremely rare. To our knowledge, there are only a few cases reported worldwide, including four cases among the 1583 NPC patients with NPC diagnosed at the Prince of Wales Hospital, Hong Kong over a 7-year period. ${ }^{3}$ The first case of NPC skin metastasis was reported in 1949 by Markson et al. ${ }^{4}$ and Pack and Booher. ${ }^{5}$ We present two additional cases of advanced NPC with multiple nodular skin metastases. 


\section{CASE REPORTS}

\section{Case 1}

A 53-year-old man presented with a six-month history of large bilateral neck masses. These were also associated with blockage of the nose, blood-stained nasal discharge, impaired hearing and tinnitus. On clinical examination, the left neck mass measured $9 \mathrm{~cm} x$ $7 \mathrm{~cm}$, the right neck mass measured $6 \mathrm{~cm} \times 7 \mathrm{~cm}$ and both were above the supraclavicular fossa, were firm and not inflamed. Rigid nasal endoscopy showed fungating masses arising from fossa of Rosenmuller on both sides. Histopathological examination of the biopsy of this mass revealed a non-keratinizing squamous cell carcinoma, undifferentiated type (WHO Type III). The computed tomography (CT) scan showed masses on bilateral sides of the nasopharynx with erosion of the floor of the left sphenoid sinus and extension into the left sphenoid sinus and left posterior ethmoid sinuses. A Diagnosis of NPC (T3, N3a Mo, Stage IVB) was made.

The patient completed radical radiotherapy to the nasopharynx and neck bilaterally. He refused concurrent chemotherapy. About one month later, he developed multiple small subcutaneous nodules all over his entire body (Figure1). Fine needle aspiration cytology (FNAC) of one of the nodules revealed metastatic carcinoma. He was given a course of palliative chemotherapy but unfortunately he succumbed to the disease a few weeks later.

\section{Case 2}

A 43-year-old woman presented with a history of multiple right neck masses of two weeks duration associated with epitaxis and tinnitus in the right ear. Clinical examination revealed multiple right neck masses, the largest measured less than $6 \mathrm{~cm}$ in diameter and was firm-to-hard in consistency. The overlying skin was not inflamed. Rigid nasoendoscopy revealed a mass confined to the nasopharynx. Histopathological examination of the biopsy of this mass revealed a non-keratinizing squamous cell carcinoma (WHO type II). The patient was then lost to follow-up and presented again one year later. At this time, the right neck nodes had already increased in size to more than $6 \mathrm{~cm}$ in diameter. The CT scan showed extension of the tumor into the right maxillary, ethmoid and sphenoidal sinuses, superiorly into the floor of the right orbit and base of skull, and inferiorly into the right parapharyngeal space, pterygoid muscles, soft palate and oropharynx. No metastases were detected at the time of this presentation. The diagnosis of nasopharyngeal carcinoma (T3, N3a, M0, stage IVB) was made.

The patient was treated with a full course of radiotherapy and concurrent chemotherapy. During the course of this treatment, she developed multiple painless skin nodules over the right upper chest and neck measuring between $0.5 \mathrm{~cm}$ to $1.0 \mathrm{~cm}$ in diameter (Figure 2). Excisional biopsies of the nodules were consistent with metastatic NPC (Figure 3). The skin metastases were treated with palliative radiotherapy with partial response. She later developed multiple bone metastases and a pathological fracture of her left femur. She succumbed to the disease approximately 20 months after initial presentation.

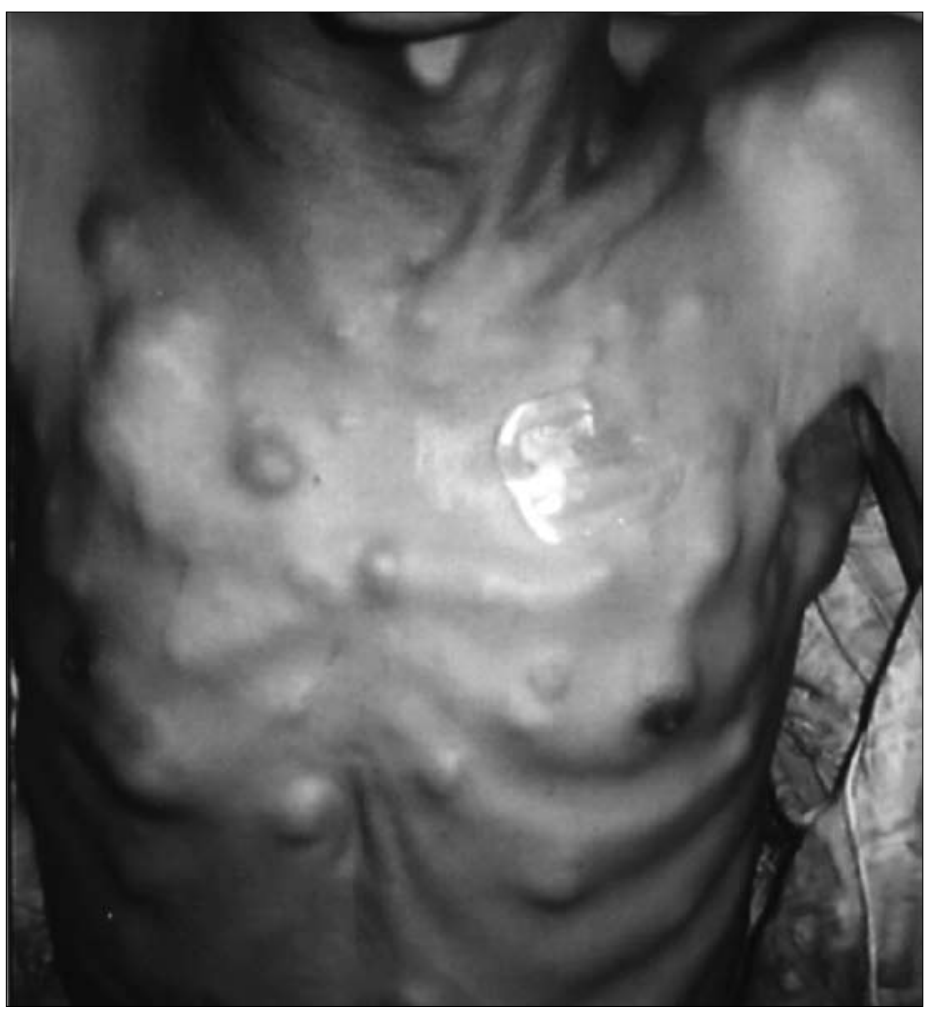

Figure 1. Nodular skin metastases on the chest

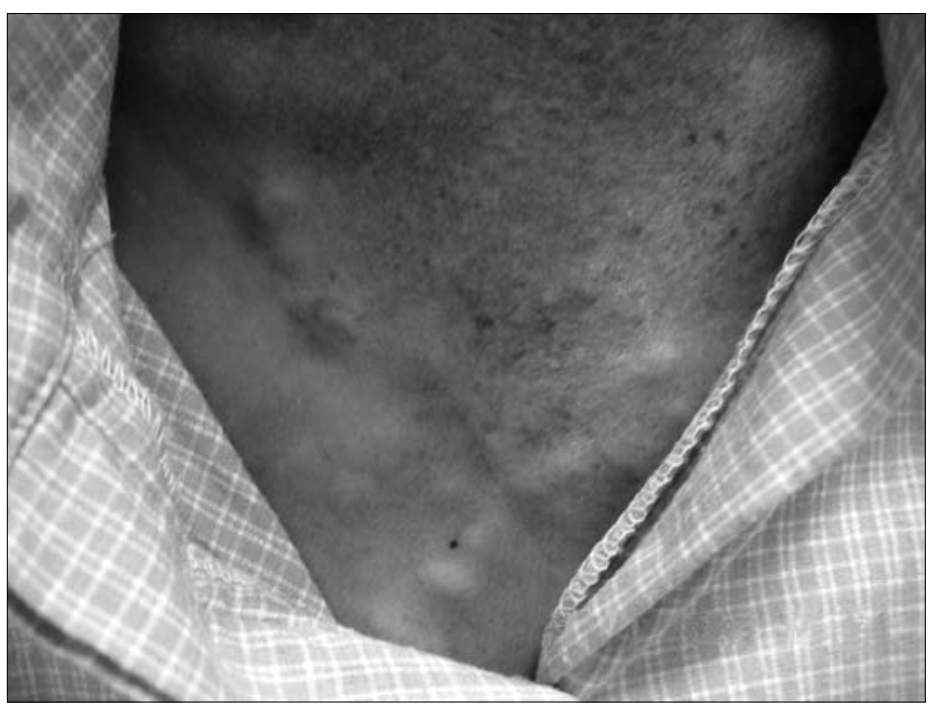

Figure 2. Nodular skin metastases on the upper chest and neck 


\section{CASE REPORTS}

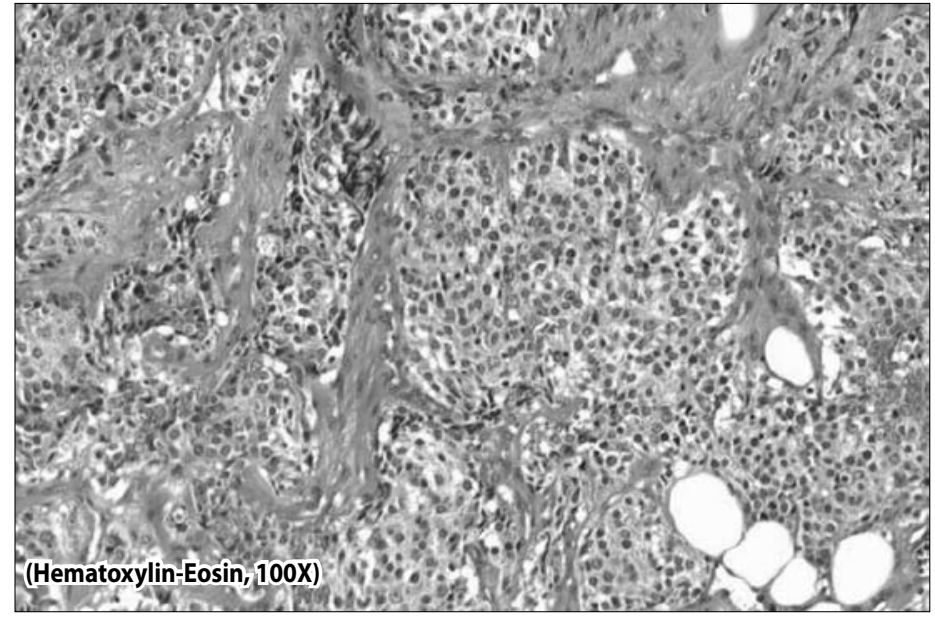

Figure 3. Sheets of malignant cell with fibrous septae in between. The malignant cells display hyperchromatic nuclei with prominent nucleoli and abundant cytoplasm.

\section{DISCUSSION}

Nasopharyngeal carcinoma (NPC) constitutes $85 \%$ of all malignant tumours of the nasopharynx. It is divided into three types according to the World Health Organization (WHO) classification; type I, keratinizing squamous carcinoma; type II, non-keratinizing carcinoma; and type III, undifferentiated carcinoma.

The diagnosis of nasopharyngeal carcinoma is based primarily on the history and physical examination. Obviously, definitive diagnosis requires a biopsy of the lesion. Molecular markers represent an exciting advance in that they can be used to generate immunotherapy that will complement conventional chemotherapy. ${ }^{6}$ Markers for nasopharyngeal carcinoma include p53, epidermal growth factor receptor (EGFR), angiogenic factors, EBV, proliferating cell nuclear antigen, $\mathrm{Ki}-67$, and c-erbB2.

The exact mechanism of how skin metastases can occur from NPC is not known. Distant skin metastases are thought to develop through hematogenous spread, whereas local metastases occur by spread via the dermal lymphatics. ${ }^{8}$ Pulmonary circulation and filtration can theoretically be bypassed via the azygous and vertebral venous system and Batson's plexus, allowing skin implantation. ${ }^{9}$

The frequency of skin metastases from internal malignancies varies from $0.7 \%$ to $9 \%$ of all patients with cancer. ${ }^{10,11}$ Common sites of skin metastases are the scalp, neck, chest, trunk, forearm, thigh, and penis. ${ }^{3}$ The major primary sources are the breast in women and the lung in men, but almost any internal cancer may metastasize to the skin. ${ }^{12}$

The metastases most commonly occur as a few solitary skin nodules. Massive and extensive nodular dissemination or diffuse dermal lymphatic infiltration as shown in both of our cases is extremely rare. Markson et al. described a 19-year-old man who presented with a transitional carcinoma of the nasopharynx that metastasized to several distant cutaneous sites. ${ }^{4}$

The mean duration between primary diagnosis and the appearance of skin metastases is 21 months with a range of 5 to 35 months following the treatment of the primary tumor. ${ }^{3}$ In addition, the diagnosis is usually associated with disseminated disease. ${ }^{3}$ In our cases, the appearance of skin metastases occurred between 6 and 15 months after the primary diagnosis and neither patient had distant visceral metastases.

Radiotherapy (RT) has been the mainstay treatment for nasopharyngeal carcinoma (NPC). Control of early-stage disease with RT alone is usually successful, but the response of locoregionally advanced NPC has been poor with frequent local relapse and distant metastasis. ${ }^{13}$

For RT, a dose of 65-75 Gray (Gy) is normally given to the primary tumour and 65-70 Gy to the enlarged cervical nodes with metastases. Radiation therapy and concurrent chemotherapy have been introduced as an alternative or standard approach for patients with advanced-stage nasopharyngeal carcinomas.

$\mathrm{RT}$ is administered according to standard fractionation and treatment of metastatic disease is based on chemotherapy. Chemotherapy is classified into three categories based on when it is delivered in relation to radiotherapy: neoadjuvant (before radiotherapy), concurrent (during radiotherapy) and adjuvant (following radiotherapy). The best chemotherapy regimen during radiotherapy has not been determined so far. To date, platinum-based regimens are the standard chemotherapy for metastatic NPC patients, and cisplatin-5-fluorouracil (5FU) combination remains the most used in first-line treatment. ${ }^{14}$ Other active chemotherapeutic agents include doxorubicin, epirubicin, bleomycin, mitoxantrone, methotrexate, and vinca alkaloids. The general consensus that chemotherapy is of limited benefit in recurrent or metastatic head and neck cancer may not be applicable to nasopharyngeal carcinoma. High objective response rates and a substantial proportion of durable complete responses have been attained in metastatic/recurrent disease. ${ }^{15}$

The duration of survival of patients with skin metastases is generally poor due to visceral metastases or advanced disease. In reported cases, this has varied between 1 and 10 months., ${ }^{3,16}$ Palliative radiotherapy, excision and different chemotherapy combinations have been used for these metastatic cases but no promising results have yet been obtained. ${ }^{17}$ Although the use of palliative chemotherapy in a patient who experiences symptoms is reasonable, the use of palliative chemotherapy in a patient without any symptoms is not as clear. The desire for prolongation of life must be balanced against the patient's 
CASE REPORTS

Philippine Journal Of Otolaryngology-Head And Neck Surgery

Vol. 25 No. 2 July - DeCEMBer 2010



quality of life, which should be the first priority. ${ }^{18}$

Nasopharyngeal carcinoma with skin metastases carries a very poor prognosis and current available treatment modalities remain ineffective in dealing with skin metastases. Early detection, diagnosis and treatment are still the best management strategy for nasopharyngeal carcinoma.

\section{REFERENCES}

1. Guigay J. Advances in nasopharyngeal carcinoma. Curr Opin Oncol. 2008 May; 20(3):264-9.

2. Altun M, Fandi A, Dupuis O, Cvitkovic E, Krajina Z, Eschwege F. Undifferentiated nasopharyngeal cancer (UCNT): Current diagnostic and therapeutic aspects. Int J Radiat Oncol Biol Phys. 1995 Jun 15; 32(3):859-77.

3. Luk NM, Yu KH, Choi CL, Yeung WK. Skin metastasis from nasopharyngeal carcinoma in four Chinese patients. Clin Exp Dermatol. 2004 Jan; 29(1):28-31.

4. Markson LS, Stoops CW, Kanter J. Metastatic transitional carcinoma of the penis simulating a chancre. Arch Derm Syphilol. 1949 Jan; 59(1):50-4.

5. Pack GT, Booher RJ. Localization of metastatic cancer by trauma. NY State J Med. 1949 Aug 1; 49(15):1839-41.

6. Agulnik M, Siu LL. State-of-the-art management of nasopharyngeal carcinoma: Current and future directions. Br J Cancer. 2005 March 14; 92(5): 799-806.

7. Mould RF, Tai THP. Nasopharyngeal carcinoma: Treatments and outcomes in the $20^{\text {th }}$ century. $\mathrm{Br}$ J Radiol 2002 Apr; 75(892):307-39.

8. Yoskovitch A, Hier MP, Okrainec A, Black MJ, Rochon L: Skin metastases in squamous cell carcinoma of the head and neck. Otolaryngol Head Neck Surg. 2001 Mar; 124(3): 248-52.

9. Batson OV. The function of the vertebral veins and their role in the spread of metastases. Ann Surg. 1940 Jul; 112(1): 138-49.

10. Brownstein MH, Helwig EB. Patterns of cutaneous metastasis. Arch Dermatol. 1972 Jun; 105(6):862-8.

11. Spencer PS, Helm TN. Skin metastases in cancer patients. Cutis. 1987 Feb; 39(2):119-21.

12. Jacyk WK, Dinkel DE, Becker GJ. Cutaneous metastases from carcinoma of the nasopharynx. $\mathrm{Br}$ J Dermatol. 1998 Aug; 139 (2):344-345.

13. Chua DT, Sham JS, Kwong DL, Au GK. Treatment outcome after radiotherapy alone for patients with Stage I-II nasopharyngeal carcinoma. Cancer. 2003 Jul; 98(1):74-80.

14. Ma BB, Chan AT. Recent perspectives in the role of chemotherapy in the management of advanced nasopharyngeal carcinoma. Cancer. 2005 Jan 1; 103(1): 22-31.

15. Fandi A, Altun M, Azli N, Armand JP, Cvitkovic E. Nasopharyngeal cancer: epidemiology, staging, and treatment. Semin Oncol. 1994 Jun; 21(3): 382-97.

16. Yücel OT, Hoşal AS, Onerci M, Sökmensüer C. Skin: An unusual site of metastases in nasopharyngeal carcinoma. Otolaryngol Head Neck Surg. 1999 Dec; 121(6):833-4.

17. Caloglu M, Uygun K, Altaner S, Uzal C, Kocak Z, Piskin S. Nasopharyngeal carcinoma with extensive nodular skin metastases: a case report. Tumori. 2006 Mar-Apr; 92(2):181-4.

18. Lin HS, Fee WE Jr. Malignant Nasopharyngeal Tumors. [article on the Internet]. Medscape@19942010 [cited 2010 Sep 28]. Available from: http://emedicine.medscape.com/article/848163 overview. 